\title{
Review on $\mathrm{g}_{-} \mathrm{C}_{3} \mathrm{~N}_{4}$ Based Fluorescence Chemical Sensor for Detection of Heavy Metal Ions
}

\author{
Abdu Hussen \\ Chemistry Department, College of Natural and Computational Sciences, Mekdela Amba University, Ethiopia.
}

DOI: http://doi.org/10.38177/AJBSR.2021.3106

Copyright: (02021 Abdu Hussen. This is an open access article distributed under the terms of the Creative Commons Attribution License, which permits unrestricted use, distribution, and reproduction in any medium, provided the original author and source are credited.

\section{ABSTRACT}

Recently, among many kinds of chemosensors, the development of fluorescent chemosensors for the sensing of environmentally toxic heavy metal ions has drawn continuous interest in fields of chemistry, materials, biological and environmental sciences due to the low cost, simple operation, high sensitivity and specificity, real-time monitoring and short response time. Upon interactions with the metal ions, the fluorescence intensity and/or fluorescence band shift of the chemosensors change and the metal ions may be detected qualitatively and quantitatively. Many methods employ techniques related to metal ion sensing. The quenching photoluminescence of carbon-based nanodots has been focused on monitoring metal ions. The employ $\mathrm{g}-\mathrm{C}_{3} \mathrm{~N}_{4}$ as fluorescence chemical sensors for heavy metal ions detection has caused increased attention largely due to its fluorescent property. Several groups have demonstrated the use of different $g-C_{3} N_{4}$ nanostructures for fluorescent metal ions sensing. Considering the properties of present chromogenic/fluorescent receptors, it seems that nanostructured $g-C_{3} N_{4}$ would be a promising alternative. The electronic structure of $g-C_{3} N_{4}$ is adjustable by coupling events of protons or metals to the surface. Therefore, the strong coordination of the nitrogen sites of $g$ - $C_{3} N_{4}$ to metal ions, causes fluorescence quenching via photoinduced electron transfer and static quenching leading to the qualitative and quantitative detection of metal ions. It has been brought into some brilliant applications, Specially for detection of $\mathrm{Cu}^{2+}, \mathrm{Fe}^{3+}, \mathrm{Hg}^{+2}$ and $\mathrm{Ag}^{+}$has been developed based on the fluorescence quenching because of the photoinduced electron transfer (PET) from $g$ - $C_{3} N_{4}$ to metal ion and also it is best alternate than other material for detection of metal ion because of its low-cost, good biocompatibility, high quantum yield, excellent stability and non-toxicity.

Keywords: Chemical sensor, Fluorescence, g-C3N4, Heavy metal, Quenching.

\section{Introduction}

The monitoring of heavy metals within the environment, drinking water, food, and biological fluids has become essential due to the raising of environmental awareness and increasingly stringent regulations for pollution control. Because of environmental pollution by heavy metal ions has increased due to the increase the use of explosive growth of industry. Heavy metals are present in all types of ecosystems. Their existence is mainly due to anthropogenic sources such as industrial and agricultural activities [1]. The waste from such activities contributes to the pollution of water bodies and soil, affecting human being. The presence of heavy metals in various samples such as soil, diet, water and natural medicinal products has frequently been reported [2]. Heavy metal ions refer metallic elements, having a relative high density, such as iron, cobalt, copper, manganese, molybdenum, zinc, mercury, plutonium and lead. Heavy metals are dangerous because they tend to bioaccumulate. Excess exposure to toxic metal ions can cause neurological, reproductive, cardiovascular, and developmental disorders [3]. Therefore finding the simple and sensitive detection method is very important.

Accurate detection of heavy metal ions is becoming increasingly important to the regulatory agencies, the regulated community and the general public. The development of very sensitive and precise instruments is a big challenge. A variety of analytical methods available to determine the trace toxic heavy metals ions, such are inductively coupled plasma mass spectrometry (ICP-MS) [4], flame atomic absorption spectrometry (FAAS) [5], graphite flame atomic absorption spectrometry (GFAAS), and inductively coupled plasma optical emission spectrometry (ICP-OES) [6]. Most of these methods showed highly selective and sensitive, but they were time-consuming and required sophisticated instrumentation or highly trained operators, which limited their own field practical application. Thus, 
there are alternative chemical sensors that have been developed for detection of heavy metal ion. These kinds of sensor are simple, low cost, highly sensitive, real time response and selective assay for heavy metal ion detection. In recent years, fluorescent probes for the detection of environmentally toxic metal cations have received extensive attention for designing and development of colorimetric or fluorescent chemosensors.

Nanotechnology-derived products have provided a wide range of material candidates that can be used to increase portability and enhance stability, selectivity and sensitivity of sensors and analytical measurement technologies. Nanotechnology is most widely used in electronics, sensing, biomaterials and catalysis [7,8]. A nanosensor is a sensor on the nanoscale, which exhibits several advantages, such as low cost, high sensitivity and high level of device integration capability. These devices that bind selectively and reversibly to the analyte with concomitant change in one or more properties of the system, such as fluorescence or color.

Among many kinds of chemosensors, the development of fluorescent chemosensors for the recognition and sensing of the biologically and environmentally toxic metal ions has drawn continuous interest in fields of chemistry, materials, biological and environmental sciences due to the low cost, simple operation, high sensitivity and specificity, real-time monitoring and short response time [9-13]. Upon interactions with the metal ions, the fluorescence intensity and/or fluorescence band shift of the chemosensors change and the metal ions may be detected qualitatively and quantitatively [14]. The development of sensitive chemosensors, which selectively recognize cations, specifically metal ions [15-19]. Many methods employ techniques related to metal ion sensing. The quenching photoluminescence of carbon-based nanodots has been focused on monitoring metal ions, such as $\mathrm{Hg}^{2+}, \mathrm{Pb}^{2+}, \mathrm{Cu}^{2+}, \mathrm{Fe}^{3+}$, and $\mathrm{Cr}^{3+}$ [20-24]. The employ $\mathrm{g}-\mathrm{C}_{3} \mathrm{~N}_{4}$ as sensors for heavy metal ions detection has caused increased attention largely due to its fluorescent property.

Graphitic carbon nitride is the most stable polymorphic analog of carbon nitride and has been the subject of great attention in the last 6 year. It is readily synthesized by calcining abundant nitrogen rich precursors such as melamine, dicyandiamide and urea. Graphitic carbon nitride $\left(\mathrm{g}-\mathrm{C}_{3} \mathrm{~N}_{4}\right)$, it can be easily combined with other compounds by ultrasonic dispersion method, deposition-precipitation method and so on. The $\mathrm{g}_{-} \mathrm{C}_{3} \mathrm{~N}_{4}$ with a band gap of $2.7 \mathrm{eV}$ is suitable to implement host/guest $\mathrm{n} / \mathrm{n}$ junction architecture with other metal oxides of appropriate flat band potentials to affect electron transfer.

In addition, because of its high nitrogen content, $\mathrm{g}-\mathrm{C}_{3} \mathrm{~N}_{4}$ can provide more active reaction sites than the other $\mathrm{CN}$ material and its lamellar structure benefits the transport of electron. $\mathrm{g}-\mathrm{C}_{3} \mathrm{~N}_{4}$ has been extensively applied in sensing [25]. It is a new type of carbon-based material with high fluorescence quantum yield. Compared with other materials, $\mathrm{g}-\mathrm{C}_{3} \mathrm{~N}_{4}$ possesses various advantages such as low-cost, good biocompatibility, high quantum yield, excellent stability and non-toxicity. The electronic band structure and band gap of $\mathrm{g}-\mathrm{C}_{3} \mathrm{~N}_{4}$ depend on the degree of condensation of the material. It was also proposed that the band gap can be tuned to lower or higher values by protonation or synthesis of inclusion complex with metal cations such as $\mathrm{Zn}^{2+}$ and $\mathrm{Fe}^{3+}[26]$.

Recently, several groups have demonstrated the use of different $g-\mathrm{C}_{3} \mathrm{~N}_{4}$ nanostructures for fluorescent heavy metal ions sensing [27-29]. Considering the properties of present chromogenic/fluorescent receptors, it seems that nanostructured $g-\mathrm{C}_{3} \mathrm{~N}_{4}$ would be a promising alternative. As in the above described, the electronic structure of 
g- $\mathrm{C}_{3} \mathrm{~N}_{4}$ is adjustable by coupling events of protons or metals to the surface. The surface functionalities of $\mathrm{g}-\mathrm{C}_{3} \mathrm{~N}_{4}$, that is, -N- site, those are important for high adsorption capacity for metal ions through chelation. Based on the unique PL property, $\mathrm{g}-\mathrm{C}_{3} \mathrm{~N}_{4}$ nanosheets have a strong response to some heavy metal ions. It has been brought into some brilliant applications.

For examples, synthesized ultrathin g C3N4 nanosheets as a rapid and ultrasensitive fluorescence sensor for detection- $\mathrm{Cu}^{2+}$ studied [28]. The other also developed a fluorescence sensor for $\mathrm{Fe}^{3+}$ and $\mathrm{Cu}^{2+}$ based on the fluore scence quenching of $\mathrm{g}-\mathrm{C}_{3} \mathrm{~N}_{4}$ [30]. Ultrathin graphitic $\mathrm{C}_{3} \mathrm{~N}_{4}$ nanofibers for selective detection of $\mathrm{Fe}^{3+}, \mathrm{Hg}^{+2}$ and $\mathrm{Ag}^{+}$are also reported [31,32]. Generally graphitic carbon nitride is an interested material for detection of heavy metal as fluorescence chemical sensor.

\section{Mechanism of Fluorescence Chemical Sensor for Heavy Metal Ions Detection}

\subsection{Introduction}

Thus, considering the increasing environmental threat posed by heavy metals and harmful chemicals, measures to remove and control these toxicants, before their emission in significant levels into the ecosystem, are urgently required. Concurrently, rapid, accurate measuring techniques and devices must be developed to monitor the progress; that is, it is essential to build a pollution prevention system wherein we can accurately and quickly assess the extent of heavy metals or harmful chemical contamination, either in the field where these pollutants are released or in the laboratory. Then, if pollutants are detected, we can take steps to prevent their release.

Therefore, sensor is a best technique for monitoring of heavy metal ions, under carefully controlled operating conditions, the analyte signal may be independent of other sample components, thus allowing the determination of the analyte without any major preliminary treatment of the sample. The role of important components in sensors is to transmit the signal without any amplification from a selective compound or from a change in a reaction. Eventually, the development of fluorescent chemosensors for the recognition and sensing of environmentally hazardous metal ions has drawn continuous interest in fields of chemistry, materials and environmental sciences due to the low cost, simple operation, high sensitivity and specificity, real-time monitoring and short response time $[9,33]$. Based upon interactions with the metal ions, the fluorescence intensity or fluorescence band shift of the chemosensors change and the metal ions may be detected qualitatively and quantitatively. Generally fluorescent chemosensors for detection transition and heavy metal ions have drawn continuous interest for many years because these ions toxic effect to human specially at high concentration.

\subsection{Chemical Sensor}

A chemical sensor can be defined as "a portable miniaturized analytical device, which can deliver real-time and on-line information in presence of specific compounds or ions in complex samples". It is a device that transforms chemical information, ranging from the concentration of a specific sample component to total composition analysis, into an analytically useful signal. The chemical information may originate from a chemical reaction of the analyte or from a physical property of the system investigated. A chemical sensor consists of a chemically selective layer, of which either the chemical or physical properties changes on interaction with an analyte, and a transducer. 
Chemical sensors form a simple integrated analytical system that integrates the reaction and measurement steps, without a break in continuity. Simple and innovative chemical sensor designs have led to the development of low cost analytical instrumentation, coupled with fast and robust analytical procedures. All chemical sensor designs comprise of two equally important parts: the receptor or recognition element and the transducer. The receptor is the area in which the chemical reaction occurs and produces a signal, which could transmit as a colour change, light emission, electrical potential, electron flow etc. The transducer responds to the recognition signal and translates the magnitude of the signal into a measure of the amount of analyte [34].

\subsection{Fluorescence Chemical Sensor}

Compounds incorporating a binding site, a fluorophore, and a mechanism for communication between the two sites are called fluorescent chemosensors. In the appropriate molecular design, fluorescent chemosensors are capable of making sensitive measurements using signals that are easy to monitor. Since these chemical tools are typically constructed using modular approaches, simple structural modifications can be made to adapt the sensor for a variety of different applications and circumstances.

Fluorescence corresponds to the relaxation of the molecule from the singlet excited state to the singlet ground state with emission of light. Fluorescence has short lifetime $\left(\sim 10^{-9} \mathrm{sec}\right)$, so that in many molecules it can compete favorably with collisional deactivation, intersystem crossing and phosphorescence. The wavelength (and thus the energy) of the light emitted is dependent on the energy gap between the ground state and the singlet excited state. It is the molecular absorption of light energy at one wavelength and its nearly instantaneous re-emission at another, usually longer wavelength. Fluorescent compounds have two characteristic spectra: An excitation spectrum (the wavelength and amount of light absorbed) and an emission spectrum (the wavelength and amount of light emitted). These spectra are often referred to as a compound's fluorescence signature or fingerprint.

This difference between absorption and fluorescence wavelength (maxima) is also known as Stokes shift and can be understood in the following way: in addition to the change of electronic structure absorption can also lead to the excitation of vibrational levels, which requires more energy or light of shorter wavelength. In some molecules like benzene, this leads to a distinct pattern (vibrational progression) in the absorption spectrum. In solution, the vibrational energy is very quickly dissipated by collisions with the solvent and the molecule adopts a new equilibrium configuration from where emission takes place. Emission can again populate excited vibrational states, this time however, in the electronic ground state. In contrast to the excitation process, the energy gaps are now smaller, leading to a shift of the fluorescence to longer wavelength [33].

\subsection{Fluorescence Chemical Sensor for Detection of Heavy Metal Ion}

Recently, fluorescence techniques have been widely used to detect changes in the concentration of heavy metal ions in the very narrow space. Typically, a system (molecule or atom) is excited by irradiation at a particular wavelength of light. The irradiated species gains energy by absorbing a photon and enters an excited state. By losing this energy, the system can relax by emitting a photon. The difference in wavelength between absorption and emission bands has been widely applied in the development of various chemical sensors since it enables analysis with high 
sensitivity as compared with a number of spectroscopic detection methods based on absorption phenomena. Thus, fluorescence spectrometry is well known to have approximately one million times more sensitivity than absorption spectroscopy. Although photo-induced electron transfer (PET), electronic energy transfer (EET), Förster resonance energy transfer (FRET), and other methods have been used to develop fluorescence-based chemical sensors, only hazardous-metal-ion-detecting sensors based on PET were described [34]. When the electron located in the LUMO relaxes to the HOMO as shown in Figure 1a below, the corresponding energy difference is converted into one of several forms, including light, chemical reaction, or heat.

If the energy is emitted as light, fluorescence or phosphorescence is observed. If the energy level of an orbital in another part of the molecule or outer substance is located between the HOMO and LUMO levels of the fluorophore, as shown in Figure $1 \mathrm{~b}$ below, electron transfer can occur from the outer orbital to the HOMO, where a hole was created upon the absorbance of light. As the HOMO is then filled, the excited electron in the LUMO is transferred to the outer orbital, emitting heat. A representative example is the case when a chemical functional group, such as an amine with a nonbonding electron pair, is attached to a fluorophore; these molecules typically exhibit very weak fluorescence and release most of the absorbed light energy as heat. This phenomenon, in which the fluorescence of a well-known fluorophore is not observed, is called fluorescence quenching by the PET mechanism.

(a)

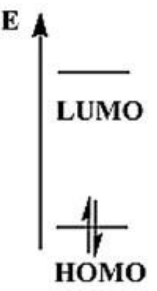

(b)

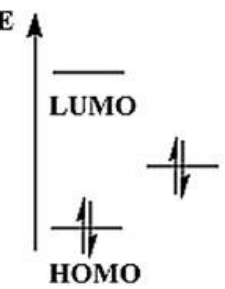

(c)

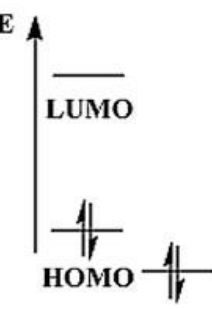

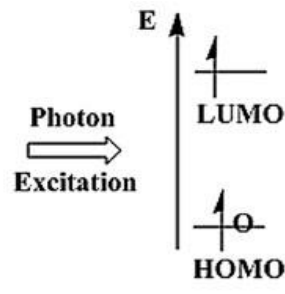
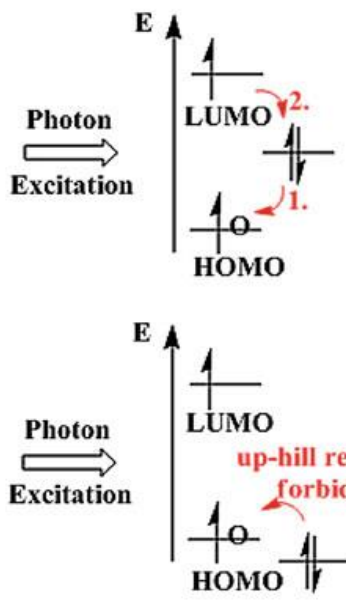

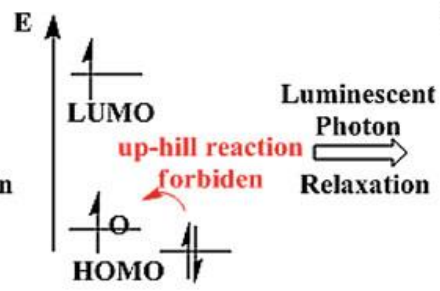

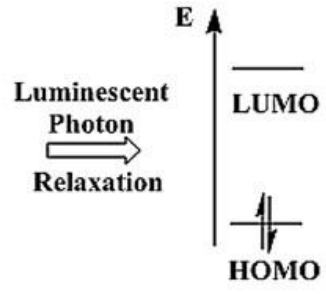
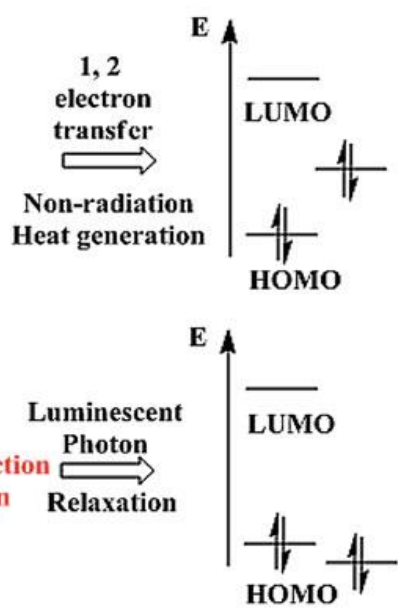

Fig.1. Schematic mechanistic representations of the relaxation process in a/ fluorescent materials, b/ fluorescent materials with an active quencher, and c/ fluorescent materials with an improper quencher

If the interaction between an analyte and the sensor molecule lowers the energy of the outer orbital below that of the HOMO, as shown in Figure 1c below, the energy level of the hole produced by absorption may be located above that of the electrons in the outer orbital. Thus, electron transfer does not occur and the fluorophore is able to emit light instead of heat. In the case of fluorescent metal ion sensors, fluorescence quenching generally does not occur when the fluorescent sensor molecule and the metal ion coexist in media. That is, we can facilitate detection by a 
"turn-on" sensor, similar to the lighting of an incandescent lamp, for which fluorescence occurs only when the analyte is present. To detect representative transition metal ions such as $\mathrm{Cr}^{3+}, \mathrm{Cu}^{2+}, \mathrm{Fe}^{3+}, \mathrm{Hg}^{2+}, \mathrm{Ag}^{+}$and $\mathrm{Hg}^{2+}$ that adversely affect the human body and environment, "turn-on" fluorescent chemical sensors based on PET through the organic connection of an amine with a light-emitter have been successfully developed [9].

Compounds incorporating a binding site, a fluorophore, and a mechanism for communication between the two sites are called fluorescent chemosensors. If the binding sites are irreversible chemical reactions, the indicators are described as fluorescent chemodosimeters. These two definitions as well as the term "fluorescent probe" have been used interchangeably and ambiguously over the past few decades therefore we have unified the area to describe them all as fluorescent chemosensors. Therefore, the development of more sensitive and selective fluorescent chemosensors, that can be used to efficiently evaluate the heavy metal ion levels in environmental and biological systems, is of great significance. Up to now, quite a lot of fluorescent chemosensors with various molecular structures and different recognition mechanisms have been developed for detection of heavy metal ions.

According to the recognition mechanism as well as the fluorescence response to heavy metal ion, the fluorescent chemosensors are classified into three categories: 'on-off', 'off-on' and ratiometric chemosensors.

\subsection{1. 'On-off' Fluorescent Chemosensors}

If the emission is efficient, the molecule may be termed a fluorophore. For example in the detection of $\mathrm{Cu}^{2+}$, its $d x^{2}-y^{2}$ orbit only has one electron, and the energy of this orbital lies between those of the HOMO and LUMO of the excited fluorophore. Thus an electron transfer or energy transfer to the $\mathrm{Cu}^{2+}$ happens and leads to a rapid nonradiative decay of the excited fluorophore. Due to the intrinsic paramagnetic nature of $\mathrm{Fe}^{3+}$ and $\mathrm{Cu}^{2+}$, quite a large number of fluorescent chemosensors for $\mathrm{Fe}^{3+}$ and $\mathrm{Cu}^{2+}$ often show a fluorescence quenching to some extent. In other word, the free chemosensors are fluorescent, and their fluorescence is quenched upon interactions with $\mathrm{Fe}^{3+}$ and $\mathrm{Cu}^{2+}$. This type of chemosensors is often called 'on-off' fluorescent chemosensor. The 'on-off' fluorescent chemosensors are classified into small molecules, supramolecules and nanomaterials according to their structural characters.

\subsubsection{Small molecular chemosensors}

To date, a variety of fluorescent chemosensors for heavy metal ion based on small molecules have been reported. Usually, a fluorescent chemosensor consists of a molecule incorporating an ion-binding site, a chromophore or fluorophore, and a mechanism for communication between the two [35]. Herein, the small molecular chemosensors are discussed according to the recognition mechanisms including intramolecular charge transfer (ICT), photoinduced electron transfer (PET), aggregation induced emission (AIE), and soon.

\section{A/ Intramolecular charge transfer (ICT) based sensors}

It is also known as photoinduced charge transfer (PCT). The PCT mechanism involves the transfer of an electron between the donor and acceptor functionalities in order to promote fluorescence [36, 37]. All the indicators have integrated ionophore, a fluorophore, as opposed to the PET indicators that have the electron donor moiety separated by spacer from the fluorophore. For this reason, with PCT indicators, the complexation of the metal ions give 
rise to alterations in electron-energy levels causing fluorescence turn-off or turn-on and a variation in emission and absorption wavelengths, depending on the type of fluorophore, metal ion, and complexation mode [36]. When a fluorophores contains an electron-donating group (often an amino group) conjugated to an electron-withdrawing group, it undergoes intramolecular charge transfer (ICT) from the donor to the acceptor upon excitation by light. The consequent change in dipole moment results in a shift that depends of the microenvironment of the fluorophore. Usually the donor and acceptor groups are conjugated in the ground state and undergo significant charge transfer in the excited state [38].

\section{B/ Photoinduced electron transfer (PET) based chemosensor}

PET is one of the most extensively adopted mechanisms for metal ion reorganization. A typical PET system often consists of an aromatic fluorophore, an aliphatic amine and a short methylene chain as the linker. When a lone electron pair is located in an orbit of the fluorophore and the energy of this orbital lies between those of the HOMO and LUMO, efficient electron transfer of one electron of the pair to the hole in the HOMO created by light absorption may occur, followed by the non- luminescent process returning to the ground state. The PET often causes a decrease in fluorescence intensity or quenching of the chemosensor [39, 40]. PET type fluorescent response does not cause any spectroscopic shifts in the emission band regarding the complexation of the metal ions.

Fluorescence in a molecule is observed when an excited electron, for instance in the lowest unoccupied molecular orbital (LUMO), goes to the highest occupied molecular orbital (HOMO), releasing the excess of energy as light. Over this, it might happen that an orbital from another part of the molecule or from another molecular entity could have energy between that of the HOMO and that of the LUMO of the fluorophore. When this "alien" orbital is full (for instance, if we have a donor group), a PET from this full orbital to the HOMO of the fluorophore can take place. A further electron transfer from the LUMO of the fluorophore to the external orbital retrieves the stable ground state. Following this sequence, fluorescence quenching occurs because the transition from the excited to the ground state takes place following a non-radiative path as illustrated in Figure 2. This process lead to a decrease of the emission intensity or no fluorescence at all.

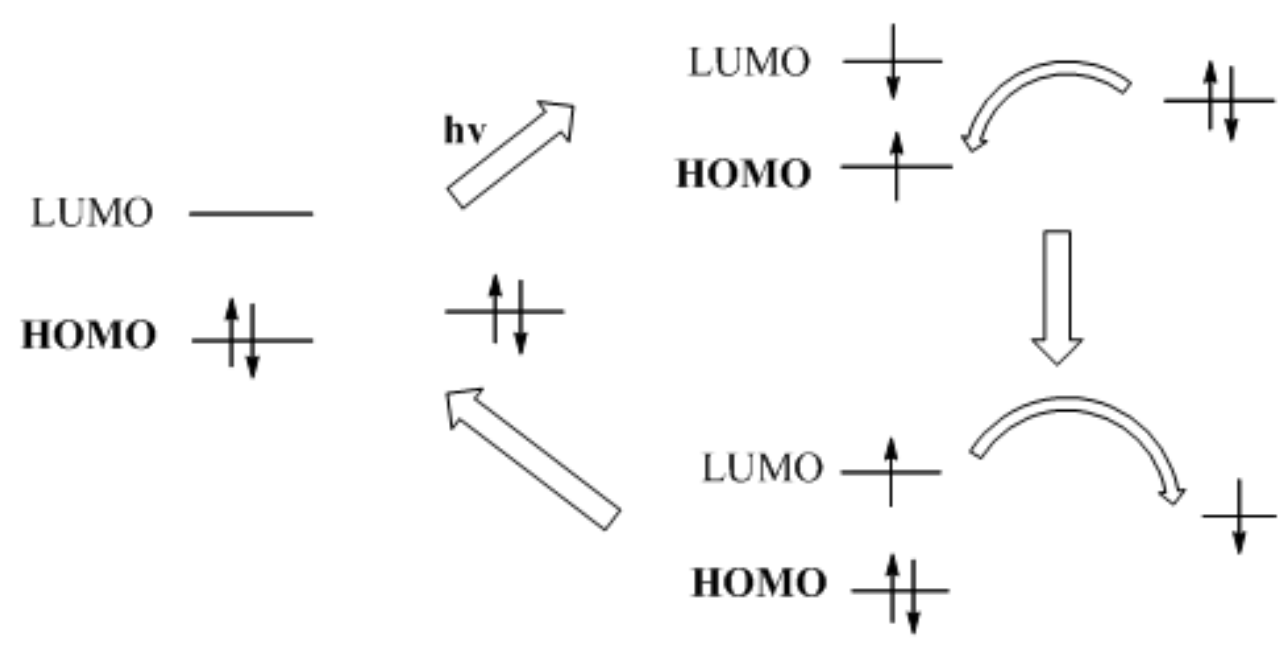

Fig.2. PET process with the participation of the HOMO and LUMO of the fluorophore and an external molecular orbital 
A similar process can take place when there is an empty orbital from another part of the molecule or from another molecular entity between both the HOMO and LUMO of the fluorophore. In this case, a PET from the excited LUMO to the empty orbital can occur, followed by a further electron transfer from this orbital to the HOMO of the fluorophore. Again, de-excitation occurs without radiation and fluorescence quenching is described in Figure 3. The design of fluorescence chemosensors tries to take advantage of such PET effects in such a way that the presence of the cation and anion should induce the appearance or the removal of energy levels between the HOMO and LUMO of the fluorophore inducing quenching or enhancement of the fluorescence emission [41].

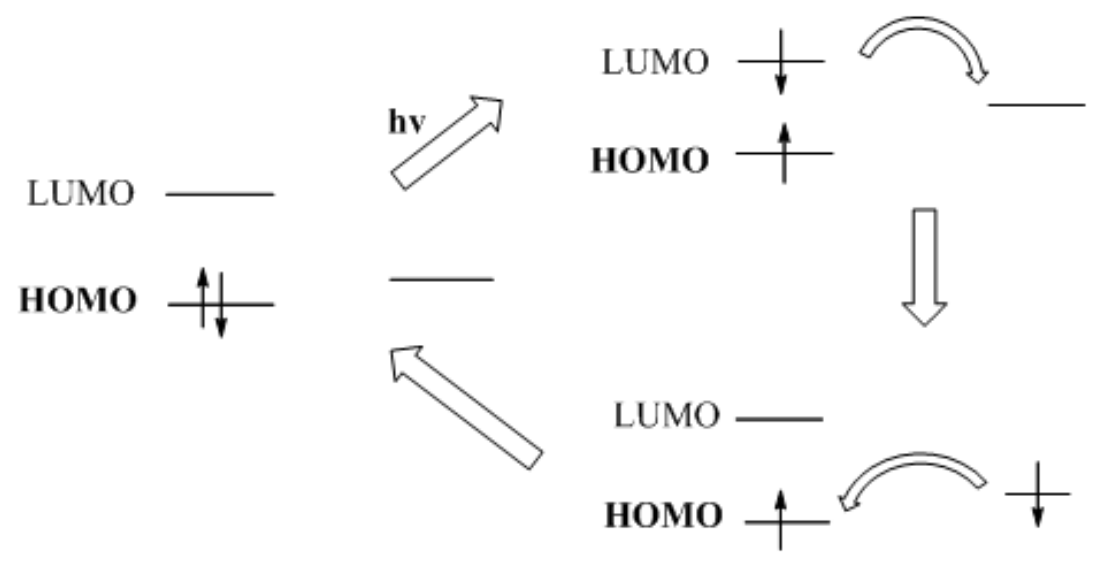

Fig.3. PET process with the participation of the HOMO and LUMO of the fluorophore and an empty external molecular orbital

\section{C/ Aggregation induced emission (AIE) based sensors}

Most fluorescent compounds suffer from the aggregation-caused quenching (ACQ) effect when dispersed in a poor solvent or fabricated into films in the solid state, which may limit their practical application. In contrast, the AIE phenomenon is precisely opposite to ACQ and provides a new strategy to broaden the application of fluorophores $[19,42]$.

\subsubsection{Supramolecular chemosensors}

Supramolecular fluorescent chemosensors are a biotic devices that produce a change in fluorescence by binding analyte by non-covalent interactions (e.g. hydrogen bonds or $\pi$-interactions for a molecule; coordination interactions for a metal ion; electrostatic interactions for an anion) [43]. In view of molecular weight, most of the supramolecular fluorescent chemosensors are small molecular chemosensors, while they have outstanding structural features different from the common small molecules. To date, a wide range of molecular structures such as crown ethers, calixarenes, helicenes, metal complexes, porphyrins, cyclodextrin, etc. have been used as host skeleton to devise various fluorescent chemosensors [44]. For example, the in fluorescent chemosensors for $\mathrm{Cu}^{2+}$ of the representative supramolecules such as perazamacrocycle, cyclodextrin and calixarene are some important one.

\subsubsection{Fluorescent nanomaterials based chemosensors}

Fluorescent nanomaterials have attracted a great deal of interest due to their low cytotoxicity, easy surface functionalization, and high price competitiveness [45]. Such kind of materials can also be used to devise various 
fluorescent chemosensors for heavy metal ion. For example, $\mathrm{g}-\mathrm{C}_{3} \mathrm{~N}_{4}$ fluorescence chemosensor for detection of heavy metal ion have been developed.

\subsubsection{Off-On Type Fluorescent Chemosensors}

Although various 'on-off' fluorescent chemosensors for heavy metal ion has been reported. For example, in the case of 'on-off' fluorescent chemosensors for $\mathrm{Fe}^{3+}$ and $\mathrm{Cu}^{2+}$ owing to the paramagnetic nature of $\mathrm{Fe}^{3+}$ and $\mathrm{Cu}^{2+}$, the 'on-off' fluorescence quenching might occur in intracellular environment by artefacts other than metal complexation and could give rise to false positive results. Thus, 'off-on' fluorescent $\mathrm{Cu}^{2+}$ chemosensors are favored over the 'on-off' ones and have been developed in recent years. Compared to the 'on-off' fluorescent chemosensor, the 'off-on' fluorescent $\mathrm{Cu}^{2+}$ chemosensor is non-fluorescent or its fluorescence is very weak in absence of $\mathrm{Cu}^{2+}$. Upon binding with $\mathrm{Cu}^{2+}$, the intensity of fluorescence increases greatly due to several mechanisms such as the chelation-enhanced fluorescence [33] and the yield of new fluorophore.

\subsubsection{Ratiometric fluorescent chemosensors}

'On-off' and 'off-on' fluorescent chemosensors are based on the changes in emission intensity at a single wave-length, which tend to be affected by a variety of factors such as the instrumental efficiency, the concentration of chemosensor, and the micro-environment. In contrast, the ratiometric fluorescent chemosensors exhibit changes in the ratio of the intensities of emission at two different wavelengths, and can be used to evaluate the analyte concentration and provide a build-in correction for environmental effects [46].

\section{5. g- $\mathrm{C}_{3} \mathrm{~N}_{4}$ Fluorescence Chemosensor for Detection of Heavy Metal Ion}

Graphitic carbon nitride, as a new form of organic polymer-like material. The $\mathrm{g}-\mathrm{C}_{3} \mathrm{~N}_{4}$ structure includes Vander Waals interactions between adjacent sheets (Figure 4 below), and with strong covalent bonding within each sheet (nanosheets of tri-s-triazine connected via tertiary amines), it has very unique characteristics. Graphitic carbon nitride is the most stable polymorphic analog of carbon nitride and has been the subject of great attention in the last 5-6 year. It is often regarded as the most stable allotrope among the carbon nitride materials under ambient conditions [47]. Non planar geometry is similar but has three 6 - membered rings attached to the C-N rings, this provides $\mathrm{sp}^{3}$ rather than $\mathrm{sp}^{2}$ hybridized bonding and results in buckling of the graphitic sheets. However, the planar g- $\mathrm{C}_{3} \mathrm{~N}_{4}$ structure is more common and in referral to $\mathrm{g}-\mathrm{C}_{3} \mathrm{~N}_{4}$ the planar structure is assumed.

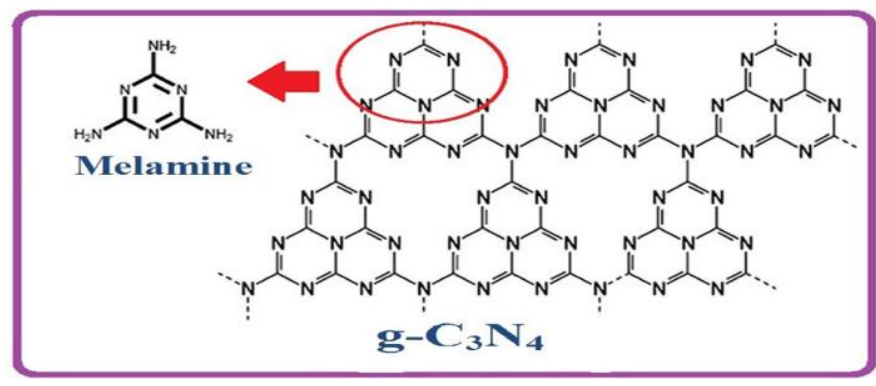

Fig.4. Structures of the graphitic carbon nitride $\left(\mathrm{g}-\mathrm{C}_{3} \mathrm{~N}_{4}\right)$

Graphitic carbon nitride is nontoxic semiconductor, exhibiting excellent photoactivity, photocatalysis and electron transport $[48,49]$. This polymer-like semiconductor material $\left(\mathrm{g}-\mathrm{C}_{3} \mathrm{~N}_{4}\right)$ is a cheap, stable material that is abundant 
and has a controllable surface, so it can be treated as a new material for environmental applications and solar energy. It is well known that the $\mathrm{g}-\mathrm{C}_{3} \mathrm{~N}_{4}$ exhibits photoluminescence (PL) properties similar to many semiconductors materials. g- $\mathrm{C}_{3} \mathrm{~N}_{4}$ emits a blue PL around $450 \mathrm{~nm}$ when dissolved in solvents under UV light irradiation due to its direct bandgap of $2.7 \mathrm{eV}$, which can be explained as the transition of the s-triazine ring [50].

Based on the unique PL property of $\mathrm{g}_{-} \mathrm{C}_{3} \mathrm{~N}_{4}, \mathrm{~g}-\mathrm{C}_{3} \mathrm{~N}_{4}$ nanosheets have a strong response to some heavy metal. For example, copper ions as turn-off chemical sensors. Besides copper ions, $\mathrm{PL}$ of $\mathrm{g}-\mathrm{C}_{3} \mathrm{~N}_{4}$ can also be quenched by other metal ions like $\mathrm{Fe}^{3+}, \mathrm{Ag}^{+}, \mathrm{Hg}^{2+}$, and $\mathrm{Cr}^{2+}$ [51-53]. The fabrication of $\mathrm{g}-\mathrm{C}_{3} \mathrm{~N}_{4}$ nanosheets for the selective detection to $\mathrm{Cu}^{2+}$ and $\mathrm{Ag}^{+}$was also studied [54]. The successfully prepared the $\mathrm{g}-\mathrm{C}_{3} \mathrm{~N}_{4}$ QDs as effective fluorescent probes for the detection of $\mathrm{Fe}^{3+}$ and $\mathrm{Cu}^{2+} \cdot \mathrm{g}_{-} \mathrm{C}_{3} \mathrm{~N}_{4}$ nanodots via a microwave-assisted approach. The produced nanodots were utilized as turn-off sensors for mercury ions with a detection limit of $0.141 \mathrm{M} . \mathrm{g}_{-} \mathrm{C}_{3} \mathrm{~N}_{4}$ are very important for detection of toxic heavy metal ion [47], some of them are below.

\subsubsection{Copper Sensing}

In 2013, Sun and co-workers developed new applications of $\mathrm{g}-\mathrm{C}_{3} \mathrm{~N}_{4}$ in fluorosensor, they prepared ultrathin $\mathrm{g}-\mathrm{C}_{3} \mathrm{~N}_{4}$ nanosheets comprised of only about three $\mathrm{C}-\mathrm{N}$ layers by ultrasonication-assisted liquid exfoliation of bulk $\mathrm{C}_{3} \mathrm{~N}_{4}$ [52]. The nanosheets exhibit high fluorescence, which might be quenched obviously by $\mathrm{Cu}^{2+}$. As shown below Figure 5, as the redox potential of $\mathrm{Cu}^{2+} / \mathrm{Cu}^{+}$lies between the conduction band (CB) and valence band (VB) of g- $\mathrm{C}_{3} \mathrm{~N}_{4}$, the photoinduced electron transfer from the $\mathrm{CB}$ to the complexed $\mathrm{Cu}^{2+}$ might occur and lead to fluorescence quenching. g- $\mathrm{C}_{3} \mathrm{~N}_{4}$ exhibited high selectivity towards $\mathrm{Cu}^{2+}$ with a detection limit as low as $0.5 \mathrm{nM}$, and has been used in determination of $\mathrm{Cu}^{2+}$ in real water samples. g- $\mathrm{C}_{3} \mathrm{~N}_{4} / \mathrm{LDH}$ films are used as a reusable luminescent sensor for $\mathrm{Cu}^{2+}[55]$.

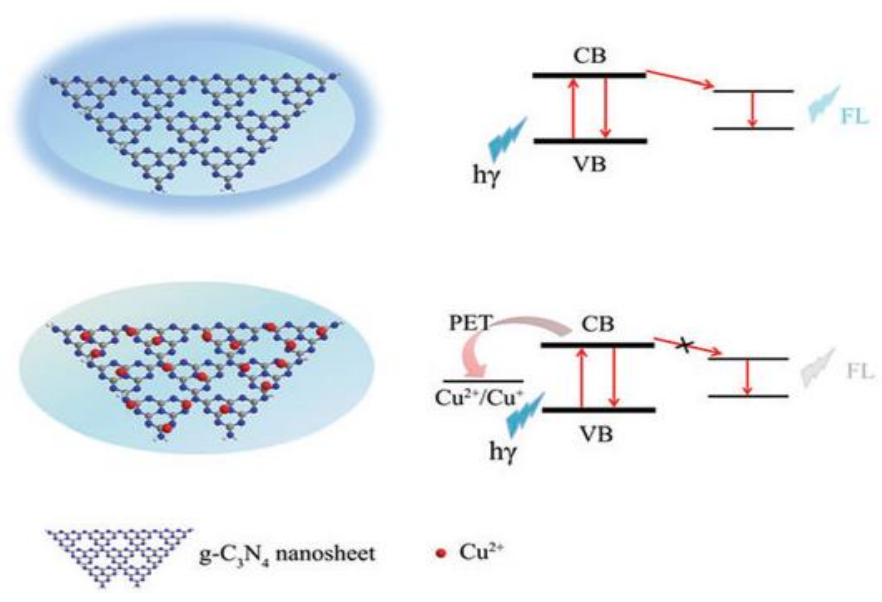

Fig.5. The quenching principle of the $\mathrm{g}-\mathrm{C}_{3} \mathrm{~N}_{4}$ nanosheets for $\mathrm{Cu}^{2+}$

\subsubsection{Mercury Sensing}

Graphitic carbon nitride also important for rapid sensing of mercury, the fluorescence quenching behaviour of the GCNNS by $\mathrm{Hg}^{2+}$ was reported as described in figure 6 [56]. The fluorescence quenching mechanism may be due to the static quenching from the formation of a stable non-fluorescent complex between the GCNNs and $\mathrm{Hg}^{2+}$. A simple and straightforward "turn-off” fluorescent sensor, where they functionalized $g-\mathrm{C}_{3} \mathrm{~N}_{4}$ sheets with DNA also 
studied. In this sensing system, $\mathrm{g}-\mathrm{C}_{3} \mathrm{~N}_{4}$ sheets worked as fluorophore, which selectively binds with the $\mathrm{Hg}^{2+}$. The fluorescence quenching was due to the electron transfer from $\mathrm{g}-\mathrm{C}_{3} \mathrm{~N}_{4}$ sheets to $\mathrm{Hg}^{2+}$ through the formed structure provided for fluorescence "turn-off" based strategy. The sensing response was measured with increasing the concentration of the $\mathrm{Hg}^{2+}$, which revealed the gradual and linear decrease in the fluorescence intensity [57, 58].

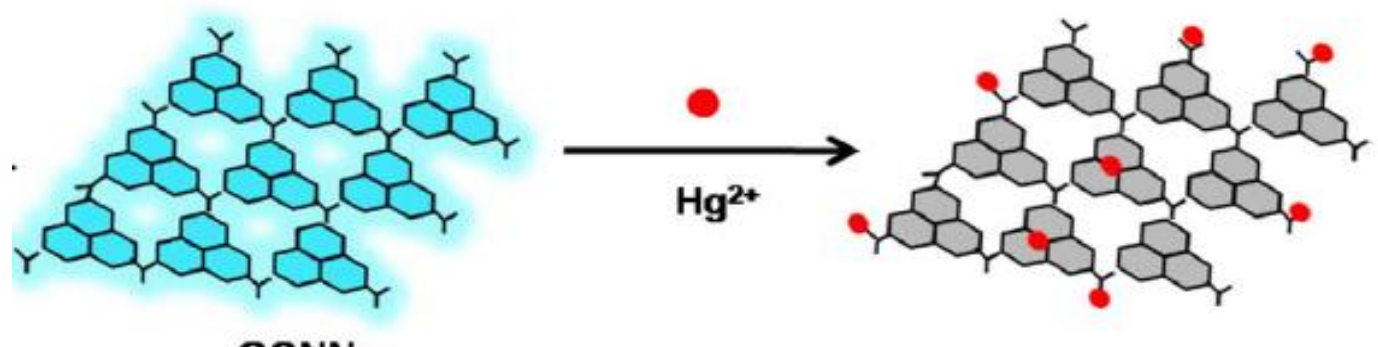

GCNNS

Fig.6. Schematic illustration of fluorescence sensing of $\mathrm{Hg}^{2+}[56]$

\subsubsection{Silver Sensing}

Graphitic carbon nitride nanosheets served as an effective fluorescent probe. As shown below Figure 7, the g- $\mathrm{C}_{3} \mathrm{~N}_{4}$ sensor can be used for sensitive detection of $\mathrm{Ag}^{+}$. The conduction band of $\mathrm{g}_{-} \mathrm{C}_{3} \mathrm{~N}_{4}$ nanosheets to $\mathrm{Ag}^{+}$led to fluorescence quenching because of the photoinduced electron transfer (PET) [32]. The potential of $g-\mathrm{C}_{3} \mathrm{~N}_{4} / \mathrm{LDH}$ films as a reusable luminescent sensor for $\mathrm{Ag}^{+}$are developed [31].

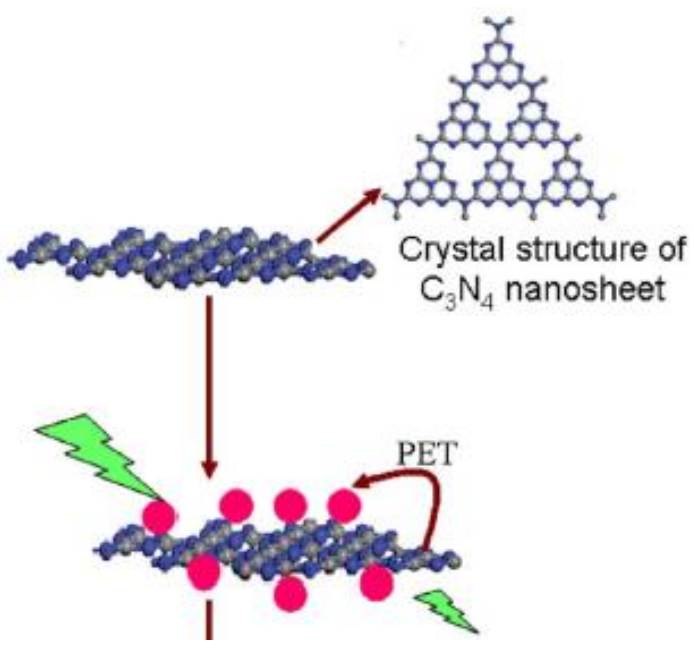

Fig.7. Schematic of $g-\mathrm{C}_{3} \mathrm{~N}_{4}$ nanosheets sensing to $\mathrm{Ag}^{+}$

\subsubsection{Iron Sensing}

Graphitic carbon nitride nanosheets also served as an effective fluorescent probe for detection of $\mathrm{Fe}^{3+}$. The introduction of $\mathrm{Fe}^{3+}$ in to the dispersion leads to an obvious decrease of fluorescence in intensity, indicating that $\mathrm{Fe}^{3+}$ can effectively quench the fluorescence of $\mathrm{g}-\mathrm{C}_{3} \mathrm{~N}_{4}$ nanofibers [59]. The binding brings $\mathrm{g}_{-} \mathrm{C}_{3} \mathrm{~N}_{4}$ and $\mathrm{Fe}^{3+}$ into close proximity with each other. Because the redox potential of $\mathrm{Fe}^{3+} / \mathrm{Fe}^{2+}$ lies between the conduction band (CB) and valence band $(\mathrm{VB})$ of $\mathrm{g}-\mathrm{C}_{3} \mathrm{~N}_{4}$, photoinduced electron transfer from the $\mathrm{CB}$ to the complexed $\mathrm{Fe}^{3+}$ occurs, leading to fluorescence quenching. 


\section{Conclusion and Future Perspective}

The finding of simple and sensitive detection method for toxic heavy metal ions is very important due to dangerous effect when they tend to bioaccumulate. Conventional analytical techniques for heavy metal ion detection have been developed, such as ICP-MS, GFAAS, FAAS and ICP-OES, but they were time-consuming and required sophisticated instrumentation or highly trained operators, which limited their on-field practical application. Thus, there are alternative fluorescence chemical sensors that has been developed for detection of heavy metal ion, Specially $\mathrm{g}_{-} \mathrm{C}_{3} \mathrm{~N}_{4}$ fluorescence chemosensor for detection of $\mathrm{Cu}^{2+}, \mathrm{Fe}^{3+}, \mathrm{Hg}^{+2}$ and $\mathrm{Ag}^{+}$has been developed ba sed on the fluorescence quenching because of the photoinduced electron transfer (PET) from $\mathrm{g}_{-} \mathrm{C}_{3} \mathrm{~N}_{4}$ to metal ion.

The research topic in fluorescent chemosensors is still in the developing stage, there are many challenges to be solved in the future. For the practical applications, an ideal chemosensor should have many features such as high sensitivity and selectivity, fast response time, ease of preparation, low toxicity, water solubility, and so on. Although many fluorescent chemosensors for heavy metal ion have been reported, they are still limited in practical applications to some extent. In addition to further improvements of these properties, other important scientific issues to pursue in this field include: to develop fluorescent chemosensors for metal ion in living organ, to study the relationship between the molecular structures and properties; and to develop heavy metal ion-promoted reaction based chemosensors.

\section{Declarations}

\section{Source of Funding}

This research did not receive any specific grant from funding agencies in the public, commercial, or not-for-profit sectors.

\section{Competing Interests Statement}

The authors declare no competing financial, professional and personal interests.

\section{Consent to participate}

Not Applicable

\section{Consent for publication}

We declare that we consented for the publication of this research work.

\section{Availability of data and material}

Authors are willing to share data and material according to the relevant needs.

\section{References}

[1] Lokhande, R. S., Singare, P. U. and Pimple, D. S. 2011. Toxicity study of heavy metals pollutants in waste water effluent samples collected from Taloja industrial estate of Mumbai, India. Resources and Environment, 1: 13-19. 
[2] Saied, S., Zahir, E. and Siddique, A. 2010. Heavy metal levels in commonly used traditional medicinal plants. Journal of Chemical Society Pak, 32 (6): 737.

[3] Kim, H.N., Ren, W.X., Kim, J.S. and Yoon, J. 2012. Fluorescent and colorimetric sensors for detection of lead, cadmium, and mercury ions. Chemical Society Review, 41: 3210-3244.

[4] Zhu, Y., Inagaki, K. and Chiba, K. 2009. Determination of Fe, Cu, Ni, and $\mathrm{Zn}$ in seawater by ID-ICP- MS after preconcentration using a syringe-driven chelating column. Journal of Analytical Atomic Spectrom, 24:

$1179-1183$.

[5] Pourreza, N. and Hoveizavi, R. 2005. Simultaneous preconcentration of $\mathrm{Cu}, \mathrm{Fe}$ and $\mathrm{Pb}$ as methylthymol blue complexes on naphthalene adsorbent and flame atomic absorption determination. Analytical Chemistry Acta, 549: $124-128$.

[6] Chaiyo, S., Chailapakul, O., Sakai, T., Teshima, N. and Siangproh, W. 2013. Highly sensitive determination of trace copper in food by adsorptive stripping voltammetry in presence of 1, 10-phenanthroline. Talanta, 108: 1-6. [7] Rae, A. 2006. Real Life Applications of Nanotechnology in Electronics. On Board Technology, 2006: 28.

[8] Duncan, T.V. 2011. Applications of nanotechnology in food packaging and food safety: Barrier materials, antimicrobials and sensors. Journal of Collide and Interface Science, 363: 1-24.

[9] Quang D.T. and Kim J.S. 2010. Fluoro- and chromogenic chemodosimeters for heavy metal ion detection in solution and biospecimens. Chemical Review, 110: 6280-6301.

[10] Kaur N. and Kumar S. 2011. Colorimetric metal ion sensors. Tetrahedron, 67: 9233-9254.

[11] Zhang J.F., Zhou Y., Yoon J. and Kim J.S. 2011. Recent progress in fluorescent and colorimetric chemosensors for detection of precious metal ions (silver, gold and platinum ions). Chemical Society Review, 40: 3416-3429.

[12] Jeong Y. and Yoo J. 2012. Recent progress on fluorescent chemosensors for metals. In. Che. Acta, 381: 2-14. [13] Sahoo, S.K., Kim, G.D. and Choi, H.J. 2016. Optical sensing of anions using C3v-symmetric tripodal receptors. Journal of Photochemistry and Photobiology C: Photochemistry Reviews, 27:30-53.

[14] Chandrasekhar V., Bag P. and Pandey M.D. 2009. Phosphorus-supported multidentate coumarin-containing fluorescence sensors for Cu2+. Tetrahedron, 65: 9876-9883.

[15] Wang H.G., Li Y.P., Fei X.L., Sun L., Zhang L.G., Zhang Z.Z., Zhang Y., Li Y.X. and Yang Q.B. 2010. Synthesis and characterization of multifunctional CdTe/Fe2O3@SiO2 core/shell nanosensors for $\mathrm{Hg} 2+$ ions detection. New Journal of Chemistry, 34: 2996-3003.

[16] Carter K.P., Young A.M. and Palmer A.E. 2014. Fluorescent sensors for measuring metal ions in living systems. Chemical Review, 114: 4564-4601.

[17] Shamsipur M. and Rajabi H.R. 2014. Pure zinc sulfide dot as highly selective luminescent probe for determination of hazardous cyanide ion. Material Science Engineering C, 36: 139-145.

[18] Lee M.H., Kim J.S. and Sessler J.L. 2015. Small molecule-based ratiometric fluorescence probes for cations, anions, and biomolecules. Chemical Society Review, 44: 4185-4191. 
[19] Yang G.H., Zhu C.Z., Du D., Zhu J.J. and Lin Y.H. 2015. Graphene-like two-dimensional layered nanomaterials: applications in biosensors and nanomedicine. Nanoscale, 7: 14217-14231.

[20] Wee S.S., Ng Y.H. and Ng S.M. 2013. Synthesis of fluorescent carbon dots via simple acid hydrolysis of bovine serum albumin and its potential as sensitive sensing probe for lead (II) ions. Talanta, 116: 71-76.

[21] Yan F., Zou Y., Wang M., Mu X., Yang N. and Chen L. 2014. Highly photoluminescent carbon dots-based fluorescent chemosensors for sensitive and selective detection of mercury ions and application of imaging in living cells. Sensor Actuators B, 192: 488-495.

[22] Zhai Y., Zhu Z., Zhu C., Ren J., Wang E. and Dong S. 2014. Multifunctional water-soluble luminescent carbon dots for imaging and Hg2+ sensing. Journal of Material Chemistry B, 2: 6995-6999.

[23] Han C., Wang R., Wang K., Xu H., Sui M., Li J. and Xu K. 2016. Highly fluorescent carbon dots as selective and sensitive "on-off-on" probes for iron (III) ion and apoferritin detection and imaging in living cells. Biosensor and Bioelectronic, 83: 229-236.

[24] Chang, M.M.F., Ginjom, I.R., Ngu-Schwemlein, M. and Ng, S.M. 2016. Synthesis of yellow fluorescent carbon dots and their application to the determination of chromium (III) with selectivity improved by $\mathrm{pH}$ tuning. Microchimica Acta, 183(6):1899-1907.

[25] Cheng, N., Tian, J., Liu, Q., Ge, C., Qusti, H.A., Abdullah, M., Asiri, M.A., Al-Youbi O.A., Xuping and Sun. 2013. Au-Nanoparticle-Loaded Graphitic Carbon Nitride Nanosheets: Green Photocatalytic Synthesis and Application toward the Degradation of Organic Pollutants. Applied Materials and Interfaces, 5: 6815-6819.

[26] Wang X. C., Chen X. F., Thomas A., Fu X. Z., Antonietti M. 2009. Advanced Material, 21: 1609 - 1612.

[27] Lee, E.Z., Jun, Y.S., Hong, W.H., Thomas, A. and Jin, M.M. 2010. Cubic mesoporous graphitic carbon (IV) nitride: An all-in-one chemosensor for selective optical sensing of metal ions. Angewandte Chemie, 122(50): 9900-9904.

[28] Tian J.Q., Liu Q., Asiri A.M., Al-Youbi A.O. and Sun X.P. 2013. Ultrathin graphitic carbon nitride nanosheet: a highly efficient fluorosensor for rapid, ultrasensitive detection of Cu2+. Analytical Chemistry, 85: 5595-5599,

[29] Huang H., Chen R., Ma J., Yan L., Zhao Y., Wang Y., Zhang W., Fan J. and Chen X. 2014. Graphitic carbon nitride solid nanofilms for selective and recyclable sensing of $\mathrm{Cu} 2+$ and $\mathrm{Ag}+$ in water and serum. Chemical Communication, 50(97): 15415-15418.

[30] Zhang W., Ma Z., Du L.P. and Li M.Y. 2014. Design strategy for photoinduced electron transfer-based small-molecule fluorescent probes of biomacromolecules. Analyst, 139: 2641-2649.

[31] Jingqi Tian, Qian Liu, Abdullah M. Asiri, Xuping Sun and Yuquan He. 2015. Ultrathin graphitic C3N4 nanofibers: Hydrolysis-driven top-down rapid synthesis and application as a novel fluorosensor for rapid, sensitive, and selective detection of Fe3+. Sensors and Actuators B, 216: 453-460

[32] Wang, S., Lu, Q., Yan, X., Yang, M., Ye, R., Du, D. and Lin, Y. 2017. “On-Off-On” fluorescence sensor based on g-C3N4 nanosheets for selective and sequential detection of Ag+ and S2. Talanta, 168:168-173.

[33] Kim J.S. and Quang D.T. 2007. Calixarene-derived fluorescent probes. Chemical Review, 107: 3780-3799. 
[34] Silva PD, Gunaratne HQ, Gunnalaugsson T, Husley AJM, McCoy CP, Rademacher JT and Rice TE. 1997. Signaling recognition events with fluorescent sensors and switches. Chemical Review, 97:1515.

[35] Czarnik A.W. 1994. Chemical communication in water using fluorescent chemosensors, Accounts of Chemical Research, 27(10),: 302-308.

[36] Formica M., Fusi V., Giorgi L. and Micheloni M. 2012. New fluorescent chemosensors for metal ions in solution. Coordination Chemistry Reviews, 256: 170-192.

[37] Liu Z., Guo Z. 2013. Metal coordination in photoluminescent sensing. Che. Soc. Rev., 42: 1568-1600.

[38] Li Y.J., Liu T.F., Liu H.B., Tian M.Z. and Li Y.L. 2014. Self-assembly of intramolecular charge-transfer compounds into functional molecular systems. Accounts of Chemical Research, 47: 1186-1198.

[39] De Silva A.P., Fox D.B., Huxley A.J.M. and Moody T.S. 2000. Combining luminescence, coordination and electron transfer for signalling purposes. Coordination Chemistry Review, 205: 41-57.

[40] Zhang S., Li J., Zeng M., Xu J., Wang X. and Hu W. 2014. Polymer nanodots of graphitic carbon nitride as effective fluorescent probes for the detection of Fe3+ and Cu2+ ions. Nanoscale, 6(8): 4157-4162.

[41] Fabbrizzi, L., Licchelli, M., Pallavicini, P., Parodi, L. and Taglietti, A. 2008. Fluorescent sensors for and with transition metals. Transition Metals in Supramolecular Chemistry, 93.

[42] Hong Y.N., Lam J.W.Y. and Tang B.Z. 2011. Aggregation-induced emission. Chem. Soc. Rev., 40: 5361-88. [43] Fabbrizzi L., Poggi A. 1995. Sensors and switches from supramolecular chemistry. Che. Soc. Rev., 24.

[44] Bell T.W. and Hext N.M. 2004. Supramolecular optical chemosensors for organic analytes, Chemical Society Review, 33: 589-598.

[45] Burns A., Ow H. and Wiesner U. 2006. Fluorescent core-shell silica nanoparticles: towards Lab on a Particle architectures for nanobiotechnology. Chemical Society Review, 35: 1028-1042.

[46] Coskun A. and Akkaya E.U. 20005. Ion sensing coupled to resonance energy transfer: a highly selective and sensitive ratiometric fluorescent chemosensor for $\mathrm{Ag}$ (I) by a modular approach. Journal of American Chemical Society, 127: 10464-10465.

[47] Cao, X., Ma, J., Lin, Y., Yao, B., Li, F., Weng, W. and Lin, X. 2015. A facile microwave-assisted fabrication of fluorescent carbon nitride quantum dots and their application in the detection of mercury ions. Spectrochimica Acta Part A: Molecular and Biomolecular Spectroscopy, 151:875-880.

[48] Dai, H., Zhang, S., Xu, G., Peng, Y., Gong, L., Li X., Li Y., Lin Y. and Chen, G. 2014. Highly photoactive heterojunction based on g-C3N4 nanosheets decorated with dendritic Zinc (II) phthalocyanine through axial coordination and its ultrasensitive enzyme free sensing of choline. RSC Advanced, 4: 58226-58230.

[49] Lu, Q., et al., 2014. Enhanced electrochemiluminescence sensor for detecting dopamine based on gold nanopower graphitic carbon nitride polymer nanosheet-polyaniline hybrids. Analyst, 139: 6556-6562.

[50] Thomas, A., Fischer, A., Goettmann, F., Antonietti, M., Müller, J.O., Schlögl, R. and Carlsson, J.M. 2008. Graphitic carbon nitride materials: variation of structure and morphology and their use as metal-free catalysts. Journal of Materials Chemistry, 18(41): 4893-4908. 
[51] Lu Y.-C., Chen J., Wang A.-J., Bao N., Feng J.-J., Wang W. and Shao L. 2015. Facile synthesis of oxygen and sulfur co-doped graphitic carbon nitride fluorescent quantum dots and their application for mercury (II) detection and bioimaging. Journal of Material Chemistry C, 3(1):73-78.

[52] Tian J., Liu Q., Asiri A.M., Sun X. and He Y. 2015. Ultrathin graphitic C3N4 nanofibers: hydrolysis-driven top-down rapid synthesis and application as a novel fluorosensor for rapid, sensitive, and selective detection of Fe3+. Sensor Actuators B, 216: 453-460.

[53] Shiravand G., Badiei A. and Ziarani G.M. 2017. Carboxyl-rich g-C3N4 nanoparticles: Synthesis, characterization and their application for selective fluorescence sensing of $\mathrm{Hg} 2+$ and $\mathrm{Fe} 3+$ in aqueous media. Sensor Actuators B, 242: 244-252.

[54] Huang H., Chen R., Ma J., Yan L., Zhao Y., Wang Y., Zhang W., Fan J. and Chen X. 2014. Graphitic carbon nitride solid nanofilms for selective and recyclable sensing of $\mathrm{Cu} 2+$ and $\mathrm{Ag}+$ in water and serum. Chemical Communication, 50: 15415-15418.

[55] Heqin Huang, Rui Chen, Jiale Ma, Li Yan, Yingqi Zhao, Yu Wang, Wenjun Zhang, Jun Fan and Xianfeng Chen. 2014. Graphitic carbon nitride solid nanofilms for selective and recyclable sensing of $\mathrm{Cu} 2+$ and $\mathrm{Ag}+$ in water and serum. Chemical Communication, 5: 15415-15418.

[56] Zhuang, Q., Sun, L. and Ni, Y. 2017. One-step synthesis of graphitic carbon nitride nanosheets with the help of melamine and its application for fluorescence detection of mercuric ions. Talanta, 164:458-462.

[57] Saleh, S.M., Ali, R. and Wolfbeis, O.S. 2011. Quenching of the luminescence of upconverting luminescent nanoparticles by heavy metal ions. Chemistry-A European Journal, 17(51):14611-14617..

[58] Zhang Y., Cui P.P., Zhang F., Feng X.T., Yang Y.Z. Liu X.G. 2016. Fluorescent probes for "off-on" highly sensitive detection of $\mathrm{Hg} 2+$ and L-cysteine based on nitrogen-doped carbon dots. Talanta, 152: 288-300.

[59] Sever, M.J., Weisser, J.T., Monahan, J., Srinivasan, S. and Wilker, J.J. 2004. Metal-mediated cross-linking in the generation of a marine-mussel adhesive. Angewandte Chemie International Edition, 43(4):448-450. 